\title{
Costochondral Joint 1
}

National Cancer Institute

\section{Source}

National Cancer Institute. Costochondral Joint 1. NCI Thesaurus. Code C102288.

The first hyaline cartilaginous joint between the ribs and costal cartilage. 